\title{
Peculiaridades da terapia trombolítica na síndrome nefrótica pediátrica: monitorização do fator anti-Xa
}

\author{
Particularities of thrombolytic therapy in pediatric nephrotic syndrome: anti-factor Xa monitoring
}

Patrícia Zambi Meirelles ${ }^{1}$, Andréia Watanabe ${ }^{2}$, Jorge David A. Carneiro ${ }^{3}$, Vera Hermina K. Koch ${ }^{4}$

\section{RESUMO}

Objetivo: Descrever a importância do tromboembolismo pulmonar (TEP) na síndrome nefrótica pediátrica e o uso da heparina de baixo peso molecular como opção terapêutica segura e eficaz.

Descrição do caso: Menino de 5,7 anos com síndrome nefrótica córtico-resistente e glomérulo-esclerose segmentar e focal foi internado devido à diarréia, distúrbios eletrolíticos e anasarca. No $11^{\circ}$ dia de internação, evoluiu com desconforto respiratório súbito, cuja investigação mostrou área de alta probabilidade de TEP na cintilografia pulmonar ventilação/perfusão e obstrução em veia jugular interna esquerda ao ultra-som doppler. Iniciado suporte ventilatório com nebulização de oxigênio e anticoagulação com enoxaparina ( $2 \mathrm{mg} / \mathrm{kg} / \mathrm{dia})$. Após seis dias, evoluiu com sintomas neurológicos compatíveis com episódio isquêmico transitório, sem alteração na tomografia computadorizada de crânio. A monitorização do fator anti-Xa no soro demonstrou nível subterapêutico e a dose de enoxaparina foi ajustada para $3 \mathrm{mg} / \mathrm{kg} / \mathrm{dia}$. O edema e os sintomas pulmonares melhoraram e o paciente recebeu alta hospitalar após 33 dias.

Comentários: Embora o TEP seja raro em crianças, a síndrome nefrótica é uma condição pró-trombótica que favorece a complicação. A heparina de baixo peso molecular pode ser considerada no tratamento e na profilaxia secundária do TEP, sendo importante monitorizar o nível sérico do fator anti-Xa para ajustar sua dose e promover tratamento seguro e eficaz.

Palavras-chave: síndrome nefrótica; tromboembolismo; anticoagulantes; criança.

\section{ABSTRACT}

Objective: Report the importance of pulmonary thromboembolism (PTE) in pediatric nephrotic syndrome and the use of low molecular weight heparin (LMWH) as an effective and secure therapeutic option.

Case description: A 5.7 year-old boy with steroidresistant nephrotic syndrome and focal segmental glomerulosclerosis was admitted to the pediatric unit with diarrhea, electrolyte disturbances and anasarca. On the $11^{\text {th }}$ day of hospital stay, he developed a sudden respiratory discomfort. Pulmonary ventilation/perfusion cintilography scan demonstrated high probability of PTE and the doppler ultrasonography showed obstruction of the left internal jugular vein. Oxygen support as well as enoxaparin, at a dose of $2 \mathrm{mg} / \mathrm{kg} /$ day, were prescribed. Six days later, the patient developed transitory cerebrovascular symptoms without alterations in the cranial CT. The serum anti-Xa factor level was lower than the therapeutic range and enoxaparin was increased to $3 \mathrm{mg} /$ $\mathrm{kg} /$ day. The edema and the pulmonary symptoms improved and the boy was discharged at the $33^{\text {rd }}$ day of stay.

Comments: Although PTE is rare in children, nephrotic syndrome is a prothrombotic condition amenable to this complication. LMWH can be considered for treatment and for secondary prophylaxis of PTE. Anti-factor Xa serum level monitoring is necessary to adjust LMWH dosage and to promote an effective and safe treatment.

Key-words: nephrotic syndrome; thromboembolism; anticoagulants; child.

\footnotetext{
${ }^{1}$ Estagiária do Instituto da Criança do Hospital das Clínicas da Faculdade de Medicina da Universidade de São Paulo (ICr/HC-FMUSP), São Paulo, SP, Brasil

${ }^{2}$ Mestre pela FMUSP, São Paulo, SP, Brasil

${ }^{3}$ Doutor pela FMUSP, professor colaborador na Disciplina de Hematologia do Departamento de Clínica Médica e da Disciplina de Pediatria Clínica do Departamento de Pediatria da FMUSP, São Paulo, SP, Brasil

${ }^{4}$ Doutor pela FMUSP, professor colaborador da Disciplina de Pediatria Clínica do Departamento de Pediatria da FMUSP e médica da Unidade de Nefrologia Pediátrica do ICr/HC-FMUSP, São Paulo, SP, Brasil
}

Endereço para correspondência:

Vera Hermina K. Koch

Rua Mangabeiras, 91/81

CEP 01233-010 - São Paulo/SP

E-mail: vkoch@terra.com.br

Recebido em: 29/12/2007

Aprovado em: 24/3/2008 


\section{Introdução}

O tromboembolismo pulmonar (TEP) é um diagnóstico incomum na faixa etária pediátrica. Entretanto, algumas situações clínicas estão associadas a uma maior incidência desta afecção, tais como utilização de cateter venoso central, doenças cardíacas, trombofilias congênitas e doença renal ${ }^{(1)}$.

A síndrome nefrótica (SN) é sabidamente associada a uma maior incidência de tromboembolismo, tanto em adultos como em crianças. A incidência de tromboembolismo nesta condição clínica varia conforme o tipo histológico da SN, uma vez que crianças com córtico-resistência apresentam maior risco do que as córtico-sensíveis ${ }^{(2)}$. As complicações tromboembólicas nos pacientes nefróticos ocorrem principalmente devido a alterações plasmáticas de proteínas envolvidas na regulação do sistema de coagulação e fibrinólise, relacionadas à presença de proteinúria e potencializadas pelo uso indiscriminado de diuréticos ${ }^{(2)}$.

Não há consenso na literatura sobre a anticoagulação como profilaxia primária na $\mathrm{SN}$, porém, seu uso em longo prazo é mandatório para aqueles pacientes que já apresentaram um episódio tromboembólico ${ }^{(3,4)}$. Dentre as possibilidades terapêuticas utilizadas atualmente, destaca-se a heparina de baixo peso molecular (HBPM), que possui algumas vantagens como meia-vida plasmática mais longa, melhor biodisponibilidade e menor incidência de efeitos colaterais, quando comparada a heparina não fracionada ${ }^{(5)}$.

Devido ao seu mecanismo de ação, a HBPM não altera o tempo de tromboplastina parcial ativada (TTPA), sugerindose que a monitorização do fator anti-Xa seja realizada em algumas situações, como alterações da função renal, grandes variações de volemia e obesidade ${ }^{(6)}$.

A descrição deste caso visa confirmar a importância da suspeita diagnóstica do TEP em crianças com SN, a utilização da HBPM como profilaxia secundária e sua eficácia e segurança por meio da adequada monitoração laboratorial com o fator anti-Xa.

\section{Descrição do caso}

Menino, cinco anos e nove meses, com diagnóstico de $\mathrm{SN}$ corticorresistente por glomeruloesclerose segmentar e focal, diagnosticada aos dois anos e 11 meses. O menor apresentou inicialmente remissão da proteinúria com esquema de prednisona associado à ciclosporina. Entretanto, após episódio de varicela, que motivou a suspensão temporária da ciclosporina, não mais apresentou resposta à terapêutica imunossupressora, seja com ciclosporina, micofenolato mofetil ou com hormônio adenocorticotrófico (ACTH). Se apresentava em anasarca, fazia uso ambulatorial de albumina em dias alternados há 14 dias e de prednisona $10 \mathrm{mg} /$ dia $(0,5 \mathrm{mg} / \mathrm{kg} / \mathrm{dia})$, em redução, quando foi internado por diarréia aguda complicada por distúrbios hidreletrolíticos (Tabela 1). No nono dia de internação, apresentou flebite em membro superior esquerdo, em local de infusão endovenosa, evoluindo com acentuação assimétrica do edema neste membro. No $11^{\circ}$ dia de internação, mostrou desconforto respiratório súbito, sudorese e alteração da ausculta pulmonar.

$\mathrm{Na}$ investigação, o ultra-som com doppler cervical evidenciou trombose extensa em veia jugular interna esquerda, com ausência de fluxo. Em radiografia simples de tórax, notou-se área de hipovascularização em região de lobo pulmonar inferior direito e derrame pleural à esquerda (Figura 1). A cintilografia pulmonar ventilação-perfusão em 24 horas mostrou área com alta probabilidade de TEP em lobo médio e inferior direito (Figura 2). O ecodopplercardiograma bidimensional não evidenciou trombose em veia cava superior, inferior ou trombos intracavitários.

O perfil da coagulação mostrou: tempo de protrombina de 14 segundos, razão normalizada internacional (RNI) de 0,88 , atividade de protrombina de $117 \%$, TTPA de 40 segundos, relação TTPA paciente/TTPA controle normal (R) de 1,47, D-dímero 600ng/mL (normal: <200), fibrinogênio de $1.131 \mathrm{mg} / \mathrm{dL}$ (normal: 155 a 395), hematócrito de 34\%, plaquetas de 365.000/dL.

Tabela 1 - Exames laboratoriais à admissão hospitalar, no $11^{\circ}$ dia de internação e na alta hospitalar

\begin{tabular}{lccc}
\hline & Admissão & $\mathbf{1 1}^{\circ}$ dia (TEP) & Alta \\
\hline Uréia $(\mathrm{mg} / \mathrm{dL})$ & 35 & 26 & 14 \\
Creatinina $(\mathrm{mg} / \mathrm{dL})$ & 0,53 & 0,32 & 0,6 \\
Sódio $(\mathrm{mEq} / \mathrm{L})$ & 124 & 130 & 139 \\
Potássio (mEq/L) & 3,0 & 5,0 & 4,4 \\
Proteína/albumina (mg/dL) & $4,4 / 0,8$ & $4,1 / 0,8$ & $4,5 / 2,3$ \\
Colesterol/TG (mg/dL) & & & $503 / 331$ \\
\hline
\end{tabular}




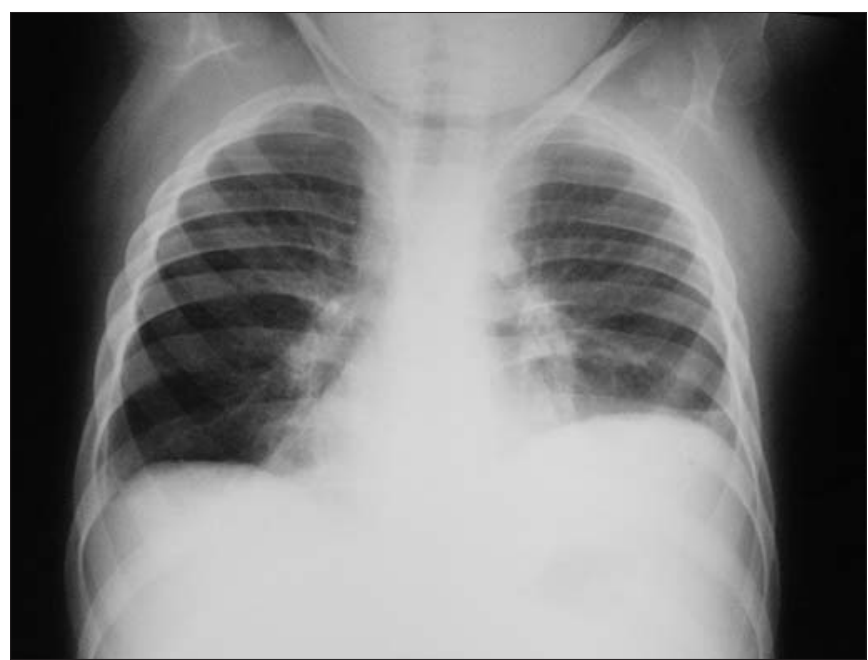

Figura 1 - Radiografia simples de tórax: área de hipovascularização em região de lobo pulmonar inferior direito e derrame pleural à esquerda.

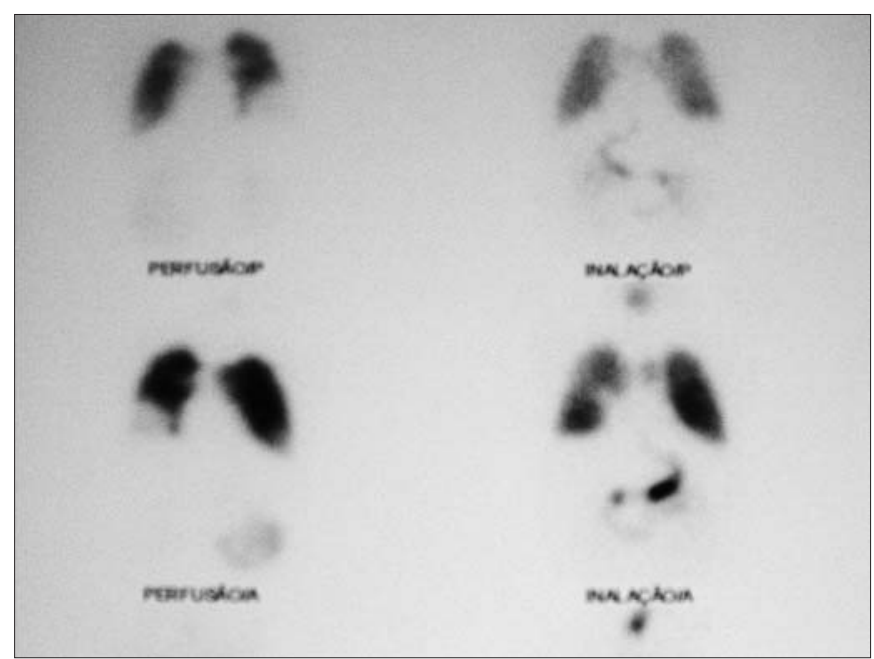

Figura 2 - Cintilografia Pulmonar Ventilação Pertusão: alta probabilidade de TEP em lobo inferior e médio à direita.

Tabela 2 - Monitorização laboratorial do tratamento com enoxaparina ao longo do tempo

\begin{tabular}{lcccc}
\hline & Início & $\mathbf{6}^{\circ}$ dia & $\mathbf{1 1}^{\circ}$ dia & $\mathbf{1 8}^{\circ}$ dia \\
\hline Dose enoxaparina $(\mathrm{mg} / \mathrm{kg} /$ dia) & - & 2 & 3 & 3 \\
Proteína total $(\mathrm{mg} / \mathrm{dL})$ & 4,4 & 3,7 & 4,3 & 4,5 \\
Albumina $(\mathrm{mg} / \mathrm{dL})$ & 0,8 & 0,9 & 1,4 & 2,3 \\
D-dímero $(\mathrm{ng} / \mathrm{mL}-$ normal $<200))$ & 600 & 800 & 1.600 & 400 \\
Anti-Xa $(\mathrm{U} / \mathrm{mL}-$ normal $0,5-0,8)$ & - & 0,2 & 0,5 & 0,5 \\
Antitrombina III $(\%-$ normal $75-125)$ & - & 60 & 86 & 93 \\
Proteinúria $(\mathrm{g} / 24 \mathrm{~h})$ & - & 5,09 & 3,16 & 6,16 \\
Fibrinogênio $(\mathrm{mg} / \mathrm{dL}-$ normal $170-400)$ & 994 & - & - & 526 \\
\hline
\end{tabular}

Iniciou-se a anticoagulação com enoxaparina, na dose de $2 \mathrm{mg} / \mathrm{kg} / \mathrm{dia}$, e suporte ventilatório por nebulização com oxigênio $5 \mathrm{~L} / \mathrm{min}$. Após seis dias de tratamento do TEP com enoxaparina, o paciente apresentou episódio súbito de apnéia e rebaixamento do nível de consciência, com rápida recuperação. Realizada tomografia computadorizada de crânio, que não mostrou alterações, sendo feita hipótese diagnóstica de episódio isquêmico transitório. Frente a um novo episódio isquêmico, em vigência de anticoagulação por via sistêmica, avaliou-se a possibilidade de anticoagulação insuficiente e dosou-se o fator anti-Xa. Este se mostrou inadequadamente baixo (Tabela 2), motivando o aumento da dose da enoxaparina para $3 \mathrm{mg} / \mathrm{kg} / \mathrm{dia}$. A Tabela 2 demonstra os valores do perfil de coagulação utilizados para monitorar a terapêutica com enoxaparina.

O paciente evoluiu com melhora do quadro pulmonar, diminuição do edema e melhora do padrão cintilográfico, recebendo alta hospitalar após 33 dias de internação, sem alteração neurológica.

\section{Discussão}

As complicações tromboembólicas são incomuns na infância, quando comparadas à população adulta ${ }^{(1)}$. O registro canadense pediátrico de trombofilia encontrou incidência de TEP de 0,86 eventos/10.000 admissões hospitalares, representando $17 \%$ dos eventos tromboembólicos em centros pediátricos terciários ${ }^{(7)}$. A incidência de TEP em crianças com $\mathrm{SN}$ varia de 1,8 a $28 \%$ e, de acordo com estudos prospectivos, a maior incidência é observada naquelas crianças com SN que realizaram estudo cintilográfico de ventilação perfusão sistematicamente, mesmo na ausência de sintomas ${ }^{(8,9)}$. Esse achado demonstra que a incidência de TEP, ainda que elevada, é subestimada na população infantil portadora de $\mathrm{SN}^{(10)}$. Os vasos mais afetados são, em ordem decrescente de importância, as veias profundas de membros inferiores, a veia cava superior, as veias renais e os vasos pulmonares. $\mathrm{O}$ envolvimento de vasos cerebrais é raro ${ }^{(2)}$.

Várias alterações homeostáticas que predispõem a um estado de hipercoagulabilidade têm sido relacio- 
nadas à SN, justificando a maior incidência de eventos tromboembólicos ${ }^{(2)}$. Dentre estas, são citadas: albuminúria, hiperfibrinogenemia, diminuição da antitrombina III (AT III), níveis elevados de dímero D e de marcadores de ativação da coagulação ${ }^{(11)}$.

A hipoalbuminemia favorece a síntese de tromboxane A2 pela plaqueta e por síntese hepática, o que leva ao desenvolvimento de hipercolesterolemia e à elevação dos fatores pró-coagulantes fibrinogênio, fibronectina, fator $\mathrm{V}$, VIII e XIII, que, em conjunto, contribuem para a ativação plaquetária ${ }^{(11)}$. O aumento de consumo de fatores prócoagulantes pode ser exacerbado pela formação de trombos intravasculares ${ }^{(12)}$. Por outro lado, a diminuição das proteínas anticoagulantes, como a AT III, o plasminogênio e a proteína $\mathrm{S}$, se dá por perda urinária, juntamente com a albuminúria ${ }^{(12,13)}$.

Dessa maneira, um grande número de fatores acaba por predispor à trombofilia, destacando-se o elevado número de plaquetas, aliado à hiperviscosidade por aumento de macroproteínas, à hipovolemia por diminuição de pressão oncótica e à dislipidemia, além de variáveis precipitantes como imobilização prolongada, uso inadvertido de diuréticos, altas doses de corticoterapia e lesão endotelial relacionada, por exemplo, à presença de cateteres vasculares ${ }^{(14)}$. Deve-se, no entanto, salientar que, apesar da comprovada tendência à trombofilia do paciente nefrótico, frente a um evento tromboembólico em criança com SN, a possibilidade de uma coagulopatia de origem genética não pode ser descartada e recomenda-se que seja investigada ${ }^{(14)}$.

No paciente nefrótico, a utilização de anticoagulação profilática não é recomendada pelos seus riscos inerentes ${ }^{(14)}$. No entanto, após o tratamento de um episódio trombótico confirmado, recomenda-se a manutenção de anticoagulação, como profilaxia secundária, por pelo menos seis meses, na forma de warfarina ${ }^{(3)}$. A anticoagulação profilática pode também ser indicada se o uso de cateteres vasculares (que deve ser, a priori, evitado) for considerado absolutamente necessário. A utilização de aspirina pode ser uma opção na terapia anticoagulante, principalmente na vigência de trombocitose $\mathrm{e}^{(14)}$.

A HBPM mostra-se uma alternativa atraente para o tratamento da fase aguda e para a terapêutica profilática do episódio trombótico ${ }^{(3)}$, com resultados comparáveis aos anticoagulantes orais, porém com menos efeitos colaterais $^{(15)}$. A HBPM consiste em fragmento da heparina não-fracionada, produzida por controle enzimático ou por despolarização. Sua ação anticoagulante depende da interação com a AT III: após a ligação da AT III com o pentassacarídeo, ocorre uma mudança de conformação da AT III, acelerando a interação da trombina com o fator $\mathrm{Xa}^{(5)}$. A HBPM apresenta como vantagens: maior meiavida, menor dependência de níveis séricos de proteínas plasmáticas, menor associação com trombocitopenia, com risco de sangramento e osteoporose quando utilizada em longo prazo, sendo sua resposta anticoagulante mais previsível. Contudo, o seu emprego deve ser rigorosamente monitorizado em pacientes com disfunção renal e grande variabilidade de volemia ${ }^{(16)}$. O controle terapêutico da HBPM é baseado no nível sérico do fator anti- $\mathrm{Xa}^{(3)}$. A dose deve ser adequada para manter o fator anti-Xa entre 0,5 a $1,0 \mathrm{U} / \mathrm{mL}$, dosado quatro a seis horas após sua aplicação subcutânea ${ }^{(4)}$.

O caso aqui descrito mostra uma criança em fase de descompensação nefrótica, com fatores predisponentes para trombofilia típicos, como hipoalbuminemia, proteinúria maciça, fibrinogenemia elevada, hipovolemia, no caso associada a processo diarréico, com cateter vascular, imobilizado no leito, confirmando que eventos tromboembólicos, incluindo o TEP, são complicações a serem suspeitadas em crianças com SN, principalmente na vigência de agravo clínico. Após a suspeita clínica e a confirmação diagnóstica, a terapia anticoagulante deve ser iniciada.

O D-dímero, freqüentemente utilizado na triagem de quadros clínicos sugestivos de trombose e que se mostrou positivo no paciente descrito, é um derivado da fibrina com alta sensibilidade e baixa especificidade para o diagnóstico de trombose ${ }^{(17)}$, sendo sua positividade não conclusiva em termos diagnósticos, principalmente no paciente nefrótico. Ksaizek et al avaliaram dez crianças, de três a dez anos de idade, em vigência de nefrose descompensada. Tais pacientes demonstraram valores significantemente mais elevados de D-dímero do que controles normais ${ }^{(18)}$. Lilova et al, em estudo retrospectivo, analisaram 447 crianças com SN, num período de 20 anos, e observaram que a hipoalbuminemia (12 a $21 \mathrm{~g} / \mathrm{L})$ e a proteinúria acima de $10 \mathrm{~g} / \mathrm{L}$ tiveram valor preditivo para o risco de tromboembolismo, assim como o uso indiscriminado de diuréticos, a hipovolemia e o estado infeccioso. Os autores, no entanto, não observaram efeito pró-trombótico significativo relacionado a outros parâmetros laboratoriais como hemoglobina, hematócrito, contagem de plaquetas, fibrinogênio e tempo de protrombina ${ }^{(2)}$. Não há descrição de um único teste, capaz de predizer com alta sensibilidade e especificidade o risco trombótico real na $\mathrm{SN}^{(14)}$. O pre- 
sente relato reforça também a necessidade de monitorizar os níveis plasmáticos da AT III, nem sempre disponíveis em nosso meio, para a utilização eficiente da HBPM.

Pode-se concluir que a SN é uma condição favorável para desenvolvimento de TEP, apesar deste ser raro na faixa etária pediátrica. A HBPM pode ser utilizada na terapêutica e na profilaxia secundária de tromboembolismo em crianças com síndrome nefrótica, sendo a monitorização do fator anti-Xa importante para adequar a dose, a fim de estabelecer tratamento seguro e eficaz.

\section{Referências bibliográficas}

1. Babyn PS, Gahunia HK, Massicotte P. Pulmonary thromboembolism in children. Pediatric Radiol 2005;35:258-74.

2. Lilova MI, Velkovski IG, Topalov IB. Thromboembolic complications in children in Bulgária (1974-1996). Pediatr Nephrol 2000;15:74-8.

3. Andrew M, Michelson AD, Bovill E, Leaker M, Massicotte MP. Guidelines for antithrombotic therapy in pediatric patients. J Pediatr 1998;132:575-88.

4. Monagle P, Chan A, Chalmers E, Michelson AD. Antithrombotic therapy in children: the Seventh ACCP Conference on Antithrombotic and Thrombolytic Therapy. Chest 2004;126 (Suppl 3):645-87S.

5. Weitz Jl. Low-molecular-weight heparins. N Engl J Med 1997;337:688-98.

6. Hirsh J, Raschke R. Heparin and low-molecular-weight heparin: the Seventh ACCP Conference of Antithrombotic and Thrombolytic Therapy. Chest 2004;126 (suppl 3):188-203S.

7. Monagle P, Adams M, Mahoney M, Ali K, Barnard D, Bernstein M et al. Outcome of pediatric thromboembolic disease: a report from the Canadian childhood trombophilia registry. Pediatr Res 2000;47:763-6.

8. Mehls O, Andrassy K, Koderisch J, Herzog U, Ritz E. Hemostasis and thromboembolism in children with nephrotic syndrome: differences from adults. J Pediatr 1987;110:862-7.

9. Hoyer PF, Gonda S, Barthels M, Krohn HP, Brodehl J. Thromboembolic complications in children with nephrotic syndrome: risk and incidence. Acta Paediatr Scand 1986;75:804-10.
10. Citak A, Emre S, Sâirin A, Bilge I, Nayir A. Hemostatic problems and thromboembolic complications in nephrotic children. Pediatr Nephrol 2000;14:138-42.

11. Schlegel N. Thromboembolic risk and complications in nephrotic children. Semin Thromb Hemost 1997;23:271-80.

12. Sagripanti A, Barsotti G. Hypercoagulability, intraglomerular coagulation, and thromboembolism in nephrotic syndrome. Nephron 1995;70:271-81.

13. Kanfer A. Coagulation factors in nephrotic syndrome. Am J Nephrol 1990;10 (Suppl 1):63-8.

14. Eddy A, Symons JM. Nephrotic syndrome in childhood. Lancet 2003;362: 629-39.

15. Yang SH, Lee CH, Ko SF, Chen JB, Chung FR, Hsu KT. The successful treatment of renal-vein thrombosis by low-molecular-weight heparin in a steroid-sensitive nephrotic patient. Nephrol Dial Transplant 2002;17:2017-9.

16. Kruse MW, Lee JJ. Retrospective evaluation of a pharmacokinetic program for adjusting enoxaparin in renal impairment. Am Heart J 2004; 148:582-9.

17. Kelly J, Rudd A, Lewis RR, Hunt BJ. Plasma D-dimers in the diagnosis of venous thromboembolism. Arch Int Med 2002;162:747-56.

18. Ksiazek J, Cichocka E, Lukasiewicz H, Teklińska E, Wyszyńska T. Molecular markers of hemostasis activation in children with nephrotic syndrome. Pediatr Pol 1995;70:1029-35. 